ARTICLE

\title{
Conserved strategies of RNA polymerase I hibernation and activation
}

\author{
Florian B. Heiss (1) ${ }^{1}$, Julia L. Daiß ${ }^{1}$, Philipp Becker $^{1} \&$ Christoph Engel (1) ${ }^{1 凶}$
}

RNA polymerase ( $\mathrm{Pol})$ I transcribes the ribosomal RNA precursor in all eukaryotes. The mechanisms 'activation by cleft contraction' and 'hibernation by dimerization' are unique to the regulation of this enzyme, but structure-function analysis is limited to baker's yeast. To understand whether regulation by such strategies is specific to this model organism or conserved among species, we solve three cryo-EM structures of Pol I from Schizosaccharomyces pombe in different functional states. Comparative analysis of structural models derived from high-resolution reconstructions shows that activation is accomplished by a conserved contraction of the active center cleft. In contrast to current beliefs, we find that dimerization of the S. pombe polymerase is also possible. This dimerization is achieved independent of the 'connector' domain but relies on two previously undescribed interfaces. Our analyses highlight the divergent nature of Pol I transcription systems from their counterparts and suggest conservation of regulatory mechanisms among organisms.

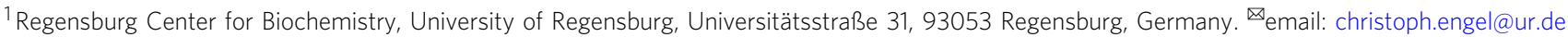


$\mathrm{T}$ ranscription of the ribosomal RNA (rRNA) precursor by RNA polymerase $(\mathrm{Pol}) \mathrm{I}$ is a prerequisite for the biosynthesis of ribosomes in all known eukaryotes ${ }^{1}$. To allow transcription initiation in baker's yeast Saccharomyces cerevisiae $(S c)$, monomeric Pol I is bound by the initiation factor Rrn3, enabling recruitment to the rDNA promoter via the heterotrimeric core factor $(\mathrm{CF})$, TBP and upstream activating factor $(\mathrm{UAF})^{2-4}$. Structures solved from Sc Pol I crystals revealed inactive polymerase dimers with widely expanded active center clefts in three similar conformations ${ }^{5-7}$, matching biochemical observations in extracts ${ }^{8}$, initial and recent Electron Microscopy studies ${ }^{9,10}$. Such dimerization relies on a 'connector' domain at the C-terminus of Pol I subunit A43. In detail, the 'stalk' subunit complex of one monomer is inserted into the 'cleft' of the other monomer from the upstream side. This allows formation of a connector a-helix in subunit A43 which attaches to the clamp core helices of subunit A190 in the second monomer. Furthermore, a C-terminal connector $\beta$-hairpin traps the lid of subunit A190, completely inactivating both polymerases ${ }^{5,6}$. Activation then requires Pol I monomerization, cleft contraction and stabilization of monomers by Rrn3. Further cleft contraction takes place upon promoter melting and Pol I interaction with the DNA/RNA hybrid ${ }^{11-13}$.

Structures of monomeric states and of actively elongating Pol I from $S c$ were solved by single particle Electron Cryo Microscopy (cryo-EM) ${ }^{14,15}$. Furthermore, differential fluorescent allele tagging demonstrated that Pol I dimerization is associated with inactivation (hibernation) under certain starvation conditions in an elegant in vivo approach ${ }^{16}$. In such experiments, deletion of the connector resulted in dimer disruption. To date, it is unclear which mechanisms and structural features of Pol I regulation are specific to $S c$ and which are conserved among organisms ${ }^{17}$.

Here, we use single particle cryo-EM to solve the structure of Pol I from Schizosaccharomyces pombe (Sp) in a monomeric (apo) and an actively elongating form at high resolution, showing that Pol I cleft contraction upon transcription activation is common to both organisms. Strikingly, we also uncover that Pol I dimerization can take place independent of the A43 connector domain in vitro and solve the cryo-EM reconstruction of such a dimer. Our results allow discussing the evolutionary conservation of structural features, hibernation and activation mechanisms.

\section{Results}

Preparation and cryo-EM of Schizosaccharomyces pombe Pol I. To study the structure of its Pol I, we generated an $S p$ strain carrying a C-terminal flag-his tag on subunit AC40. Purification using established protocols ${ }^{5}$ yielded pure Pol I that shows protein bands for all 14 subunits (Supplementary Fig. 1). Sp Pol I is active in elongation and cleavage of an RNA primer from synthetic constructs in vitro similar to Sc Pol I (Supplementary Fig. 2). To determine the structure of a monomer, we stabilized Pol I by crosslinking with BS3, followed by quenching and size exclusion chromatography (Methods). In an independent experiment, we established an $S p$ Pol I elongation complex (EC) in vitro similar to its $S c$ counterpart ${ }^{15}$. Using three locked nucleic acid (LNA) bases at the $3^{\prime}$ end of the RNA primer reduced sample heterogeneity by preventing transcript cleavage via subunit A12.2 (Methods). Two cryo-EM datasets were collected on a Titan Krios Electron Microscope equipped with Falcon III direct electron detector: one from non-crosslinked, LNA-containing EC particles and one from BS3-crosslinked Pol I, both following size exclusion chromatography (Supplementary Table 1).

Pol I architecture is conserved but sub-complex occupancy varies among organisms. The cryo-EM densities reveal secondary structure elements for the entire polymerase and side chain orientations in many regions (Fig. 1; Supplementary Figs. 1-3). The architecture of Sc and Sp Pol I is similar, despite poor overall sequence identity (Supplementary Fig. 4, Supplementary Table 2). Both, EC and monomeric Pol I, lack cryo-EM density for the A49/A34.5 heterodimer, indicating that the subcomplex is flexible or was lost during grid preparation (Fig. 1). The presence of the A49/A34.5 subunit-complex in Pol I preparations was confirmed by Coomassie-stained SDS-PAGE (Supplementary Fig. 1a) and mass-spectrometry analysis.

Differences between $S c$ and $S p$ Pol I include the lack of density for the Pol-I-specific helix $\alpha 0$ in subunit Rpb6 and insertions in the 'Dock' and 'Foot' domains of $S p$ subunit A190. Helix a0 forms only in Sc Pol I and was presumed to strengthen stalk attachment compared to Pol $\mathrm{II}^{5,6}$. A divergent region (residues 549-559) and an insertion (residues 591-600) in the 'external 2' domain of $S p$ subunit A135 are adjacent to the lobe-region responsible for tight association of the A49/34.5 sub-complex ${ }^{18}$ and may be responsible for its reduced affinity (compare Supplementary Figs. 3b and 4). Poor stalk density is observed, indicating a high degree of flexibility, similar to human Pol $\mathrm{II}^{19}$ and potentially linked to a lack of Rpb6 a0 formation. Nevertheless, fitting of a stalk homology model created from the crystal structure of the Sc A14/A43 complex ${ }^{20}$ was possible. In addition, local resolution is reduced in the jaw regions and the tip of the clamp domain of subunit A190 in Sp Pol I EC and monomer reconstructions, also indicating a high degree of flexibility (Supplementary Figs. 1 and 2). The C-terminal domain of subunit A12.2 is mostly flexible in ECs and monomers, although low-resolution density in the Sp EC (Supplementary Fig. 3a) may indicate a position outside subunit $\mathrm{A} 135$ as observed in some $\mathrm{Sc}$ $\mathrm{ECs}^{21}$.

$S p$ Pol I contains an expander element that is flexible in ECs. In ECs, we observe density for DNA and RNA, allowing us to build and refine a model for 25 template bases, 11 non-template bases and 7 RNA bases (Fig. 1d). Interactions with Pol I, positioning in the cleft and relative orientation of the scaffold are similar to Sc structures $^{14,15,21}$. We do, however, observe a density that we attribute to the 'expander' ('DNA-mimicking') element in Pol I monomers (Fig. 1b). This density is located between the 'protrusion' domain of subunit A135 and the 'clamp core' region in subunit A190 on the upstream region of the active center cleft and appears to prevent unspecific DNA binding. Due to flexible connections to the 'jaw' domain of subunit A190, we refrained from modeling the exact residues of the section in the $S p$ monomer. Location of presumed expander density in the cleft is comparable to that observed in Sc Pol I dimers (Supplementary Fig. 3d), while the element is flexible in Sc Pol I monomers.

Sp Pol I dimerizes in vitro independent of the 'connector' domain. Surprisingly, we observed many dimer-particles in the monomer dataset (Supplementary Fig. 1). As described above, Pol I subunit A43 does not contain a connector element in $S p$ and therefore should be unable to form dimers, as inferred from connector deletions in $S c^{16}$. Previous studies described that $S c$ Pol I dimers and monomers are in equilibrium in solution. Buffer conditions ${ }^{8}$ and concentration of highly purified Pol $\mathrm{I}^{5}$ can shift this equilibrium in vitro. To test whether dimerization is possible in solution or may result from chemical crosslinking, we carried out analytical size exclusion chromatography (SEC) in different buffers and in-solution dynamic light scattering (DLS) at different concentrations of $S p$ Pol I. A shift to earlier SEC elution volumes indicates an increased particle size under high-salt conditions (Fig. 2a). Negative stain EM analysis of SEC peak fractions revealed the presence of dimeric particles independent of the 

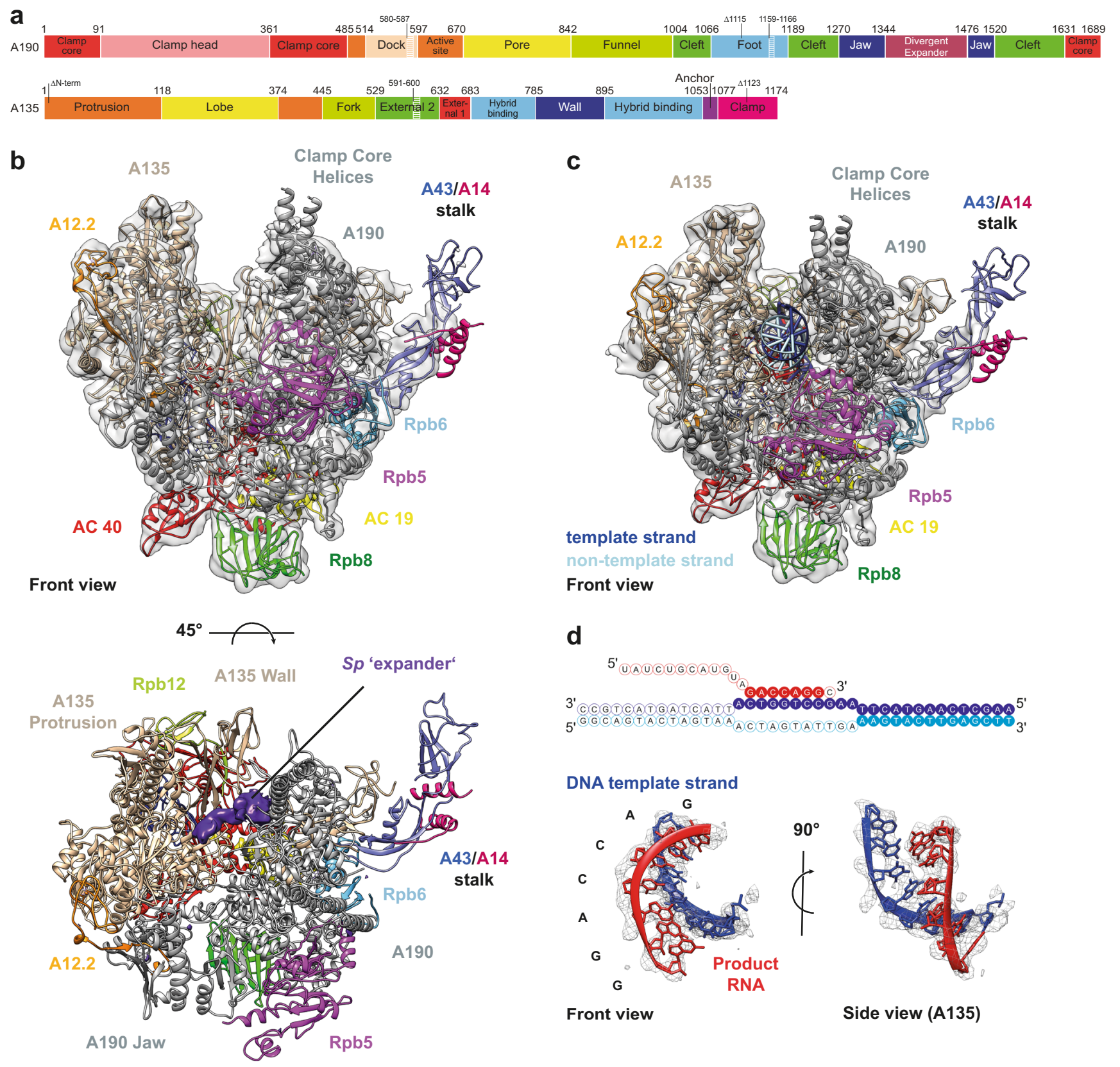

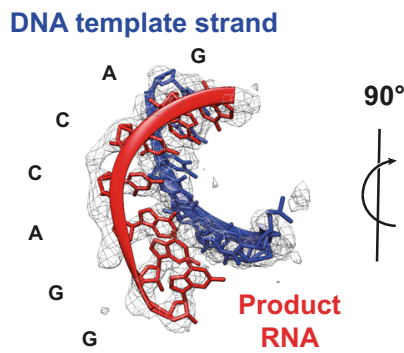

Front view

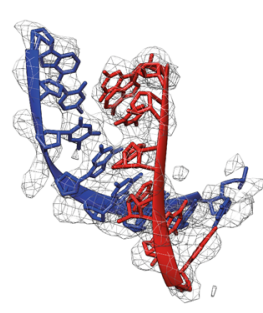

Side view (A135)

Fig. 1 Structure of monomeric and elongating S. pombe Pol I. a Sub-domain architecture of the Pol I subunits A190 and A135 in Sp. Insertion/Deletion of more than five residues compared to Sc are highlighted. b Cryo-EM reconstruction of monomeric Sp Pol I. Transparent, unsharpened cryo-EM density overlaid with ribbon model shows 12 subunits but lacks the A49/A34.5 subcomplex. The cleft is expanded and density (purple, space-filling) indicates the location of the 'expander' element. c Cryo-EM reconstruction of an Sp Pol I Elongation complex. The cleft is contracted and the expander is displaced by the hybrid. $\mathbf{d}$ Schematic representation of the artificial bubble construct used to establish an EC. Nucleotide bases included in the EC-density are highlighted. A model of the template-DNA/product-RNA hybrid overlaid with sharpened EC density is shown, the 3' nucleotide of the RNA is present but shows poor density and was thus not modeled.

buffer (Supplementary Fig. 5). In a high-salt buffer, about 32\% of identified particles ( $49 \%$ of Pol I molecules) appear to be in a dimeric state as indicated by unsupervised 2D-classification. In contrast, dimer-prevalence is below $2 \%$ of particles in low-salt conditions. In line with this, DLS using the Prometheus Panta technology (Nanotemper) indicates an increase of hydrodynamic particle radius with increasing $S p$ Pol I concentration (Fig. 2b). Taken together, we concluded that reversible, connectorindependent dimerization of $S p$ Pol I is possible. To understand the underlying molecular principles, we reconstructed the dimer architecture from its cryo-EM density.
Inactive $S p$ Pol I dimers arrange in a divergent architecture. A three-dimensional reconstruction of $S p$ Pol I dimers at $4.5 \AA$ resolution was obtained in C2 symmetry (Fig. 3; Supplementary Table 1). Unambiguous placement of two $S p$ Pol I monomers and rigid body fitting of subdomains yielded a model of the $S p$ Pol I dimer. Whereas establishment of Pol I dimers depends on stalkinsertion into the cleft of a second monomer in $S c$, the $S p$ dimers form by interaction of the stalk with the protrusion domain of subunit A135 in the other monomer (Fig. 3). A second interface forms between the 'dock domain' in subunit A190 of one monomer (including the $S p$ specific insertion of residues 580-587 
a

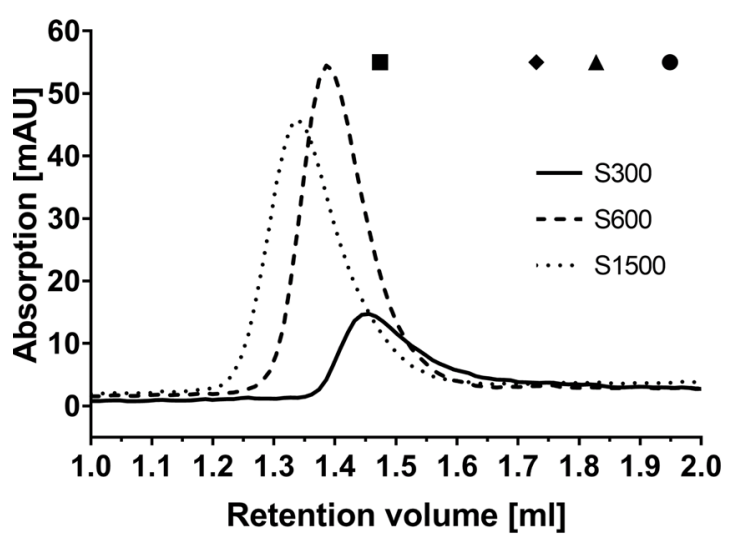

- Tyroglobulin (669 kDa) $\mathbf{S 1 5 0 0} \mathbf{5 6 0 0} \mathbf{S 3 0 0}$ $1.479 \quad 1.470 \quad 1.472$

$\Delta$ Albumin $(66.5 \mathrm{kDa})$

$\mathbf{S 1 5 0 0} \mathbf{S 6 0 0} \mathbf{S 3 0 0}$

$1.8311 .821 \quad 1.831$

b

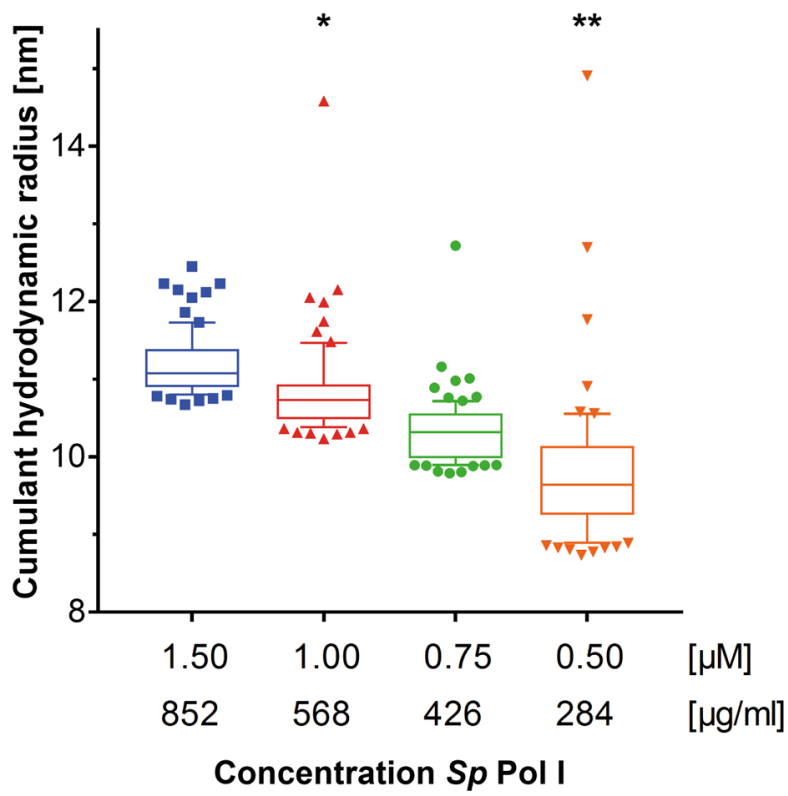

Fig. 2 Sp Pol I can dimerize in vitro dependent on protein concentration and buffer condition. a Analytical Size Exclusion Chromatography (SEC) of Sp Pol I in buffers containing 1500 mM (S1500; dotted), 600 mM (S600; dashed) or 300 mM (S300; continuous) Potassium Acetate. The main peak $\left(A_{280 n m}\right)$ elutes at higher apparent molecular weight in the high salt buffer. The average elution peaks of reference proteins are indicated: black square - Tyroglobulin; Black diamond - B-Amylase; Black triangle - Albumin; Black dot -

Carboanhydrase (exact retention volumes at the bottom). Compare Sup. Fig. 5 for negative-stain EM characterization of S300 and S1500 peak fractions and methods for details. b Dynamic Light Scattering (DLS) analysis of Sp Pol I particles (S600 buffer) measured using the Panta technology (Methods). Each mark represents an individual measurement $(n=80$ for each Pol I concentration). At higher Pol I concentration, the hydrodynamic radius of particles in solution increases, indicating a shift of equilibrium towards dimerization. The boxes show the median (horizontal line inside box) and span from the $25^{\text {th }}$ to the $75^{\text {th }}$ percentiles, the whiskers reach up to the $90^{\text {th }}$ and down to the $10^{\text {th }}$ percentile, measurements beyond these are shown as individual points ( ${ }^{\star}$ one outlier at $32.72 \mathrm{~nm}$ is not displayed; ** two outliers at $19.98 \mathrm{~nm}$ and $21.04 \mathrm{~nm}$ are not displayed). and the Pol I specific helix a12a) with the wall subdomain of subunit A135, subunit AC40 and the common subunit Rpb12 of the second monomer. These two interfaces allow a tight association via the establishment of contacts between both Pol I upstream-faces independent of the connector element but utilizing organism-specific regions. Low-resolution density on the lobe of subunit A135 in both molecules in the dimers may indicate the presence of A49/A34.5 (Supplementary Fig. 6). This is in line with the suggestion that dissociation of this subcomplex is independent of Pol I inactivation in $S p^{22}$.

Cleft contraction is common to active Pol I. Comparative structural modeling reveals that, similar to Sc Pol I reconstructions, the cleft is extended in monomers and contracted in ECs (Fig. 4; Supplementary Movie 1). Consequently, the 'bridge' helix of subunit A190 is mostly disordered in monomers (poor density despite higher overall resolution) and forms a well-structured helix in ECs (Supplementary Fig. 3e). The 'hinges' identified by comparing Pol II structures with Pol I dimers in crystals ${ }^{5,6,17}$ are similar for $S p$ Pol I cleft modulation. Thus, polymerase activation by contraction is conserved between $S c$ and $S p$. Further cleft expansion in $S c$ Pol I dimers likely results from mutual stalk-cleft insertion (Fig. 4b) and is not observed in Sp Pol I dimers (Fig. 4c; Supplementary Movie 2). Despite this divergence, functional importance is apparently conserved: Both molecules in a dimer are likely unable to initiate transcription since binding of the initiation factor Rrn3 to the stalk-dock region is blocked by the neighboring monomer. This Rrn3-binding is necessary for promoter recruitment in $S c^{10,16,23-25}$, and likely conserved in $S p^{22,26,27 .}$

\section{Discussion}

In this work, we described three single particle cryo-EM reconstructions of $S p$ Pol I, representing the only structures of this enzyme from an organism other than $S c$ to date. Availability of a second in vitro transcription system allows cross-validation of structural and functional investigations in future studies. Whereas we find that the general architecture of Pol I is conserved, structural details vary among organisms.

In contrast to $S c$, density for the expander element was observed in Sp Pol I monomers, hinting at an additional possibility to prevent unspecific DNA-binding to Pol I monomers. Absence of cryo-EM density for the A49/A34.5 heterodimer may result from a loss of the subcomplex due to stress on the airbuffer-interface during freezing ${ }^{28}$, a flexible association in $S p$, or may indicate functional relevance. The A49/A34.5 subunitcomplex plays an important role in Pol I initiation by stabilizing the non-template strand during promoterDNA melting and may support promoter escape in $S c^{12,29}$. The subcomplex shows similarities to initiation factors TFIIF and TFIIE in the Pol II transcription system, as suggested from homology to crystal structures and native mass spectrometry analysis ${ }^{30}$. However, the A49/A34.5 subcomplex has also been described as important for Pol I elongation ${ }^{20,31,32}$ and is constantly attached to the Pol I core throughout elongation in vivo ${ }^{33}$. Dissociation of the subcomplex from Sc Pol I was observed under specific experimental conditions in vitro ${ }^{20}$ or in ECs established using a nucleotide analogon $^{21}$. Notably, the EC scaffold used in this study contains modified RNA which may lead to the loss of A49/A34.5 $5^{21}$, even though this was not the case in an Sc Pol I EC structure determined with the identical scaffold ${ }^{15}$. Taken together, it can be speculated that the A49/A34.5 subcomplex not only carries out multiple functions in Pol I initiation and elongation (as recently reviewed ${ }^{34}$ ), but also that these roles may vary in importance 
a

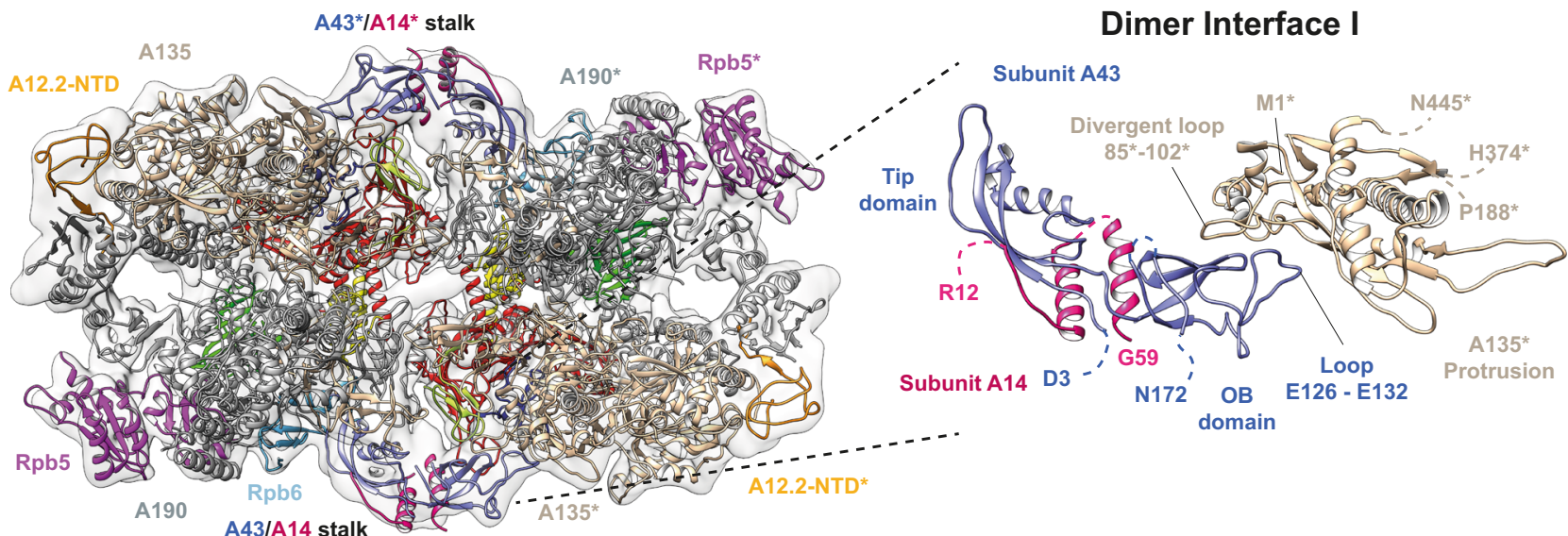

Monomer A

Monomer B

Dimer Interface II
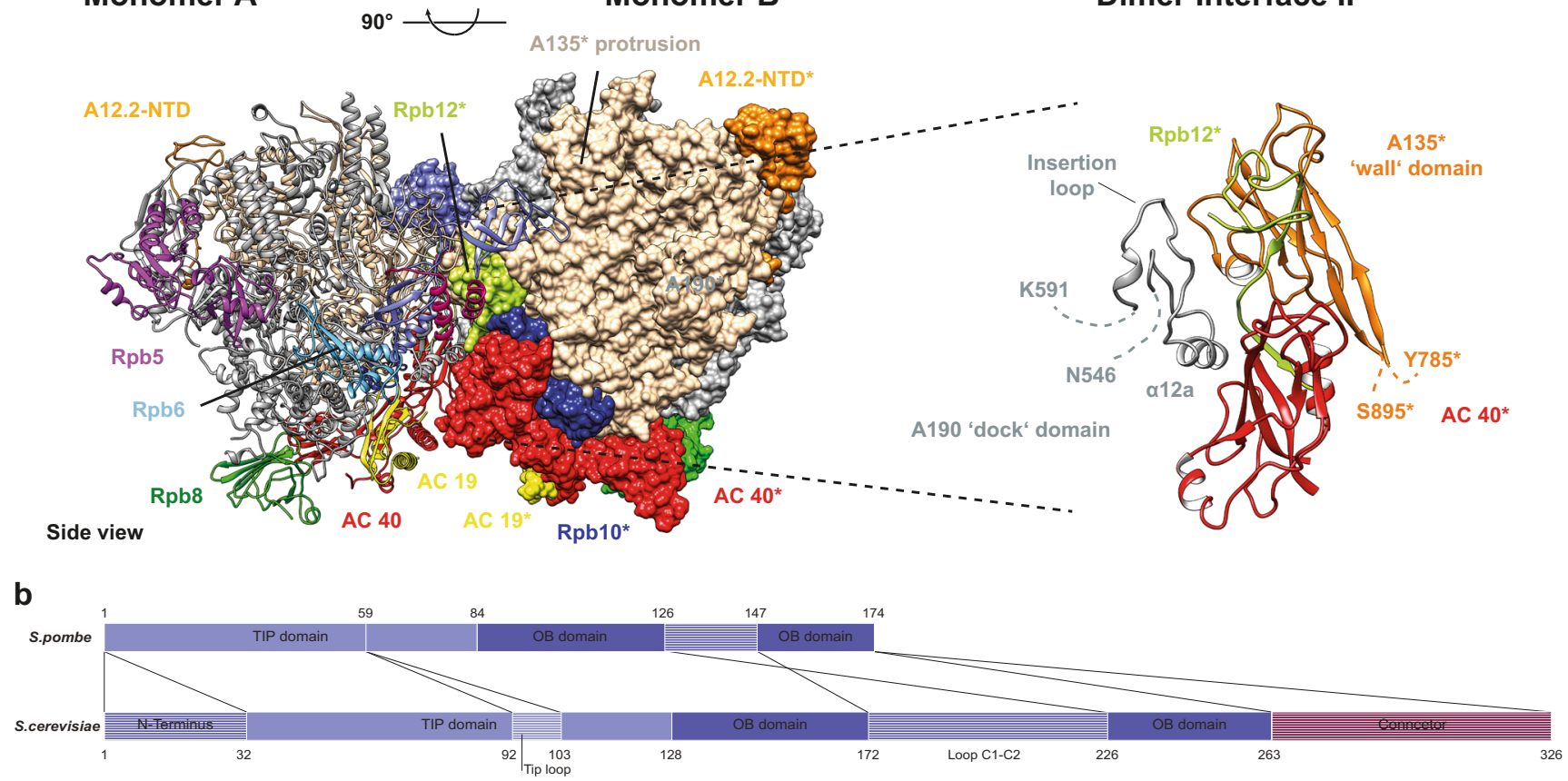

Fig. 3 The architecture of $\boldsymbol{S p}$ Pol I dimers diverges from its Sc counterpart. a Cryo-EM reconstruction of dimeric Sp Pol I (transparent, unsharpened density). Dimers form by interaction of two monomers via two interfaces (right) on their upstream faces. Subunits of monomer B (ribbon in top panel, spacefilling at the bottom) marked by asterisks. Interfaces are highlighted on the right and are largely composed of Sp-specific segments such as divergent loops in the protrusion of subunit A135, the OB-fold of subunit A43 and the dock domain of subunit A190. Some additional density in the back-region of both monomer clefts likely relates to the expander element. b Sub-domain architecture comparison of Pol I subunit A43 between Sp and Sc reveals the lack of a connector and a divergent C1-C2 loop. This loop is located at the distal end of the stalk sub-complex and involved in dimer-formation (compare panel a) by contacting the protrusion of the neighboring polymerase.

among organisms, even though functional complementation of $S c$ and Sp A49/A34.5 was previously shown ${ }^{22,35}$.

Comparison of Pol I cleft expansion states showed that transcription activation by active center cleft contraction is conserved in $S c$ and $S p$. This contraction distinguishes the Pol I transcription system from its Pol II/III counterparts ${ }^{2,36}$. Strikingly, well-defined dimers are present in Sp Pol I preparations that form independent of the A43 connector domain, which is required for dimerization in $S c^{16}$. Dimer prevalence in vitro is influenced by buffer conditions and Pol I concentration in a dynamic equilibrium in both organisms and not an artefact of chemical crosslinking or crystallization. We find that different organism-specific regions are involved in formation of inactive dimers in both, Sp and Sc Pol I, occluding the binding site for Rrn3 in both organisms. Hence, conserved Rrn3-association ${ }^{23,37,38}$ can likely stabilize monomers as required for activation ${ }^{39}$. Regulated binding of $\mathrm{Rrn}^{40-43}$ then allows promoter recruitment and DNA-melting to take place ${ }^{29,44}$.
Apparently, dimerization of Pol I molecules is possible but involves divergent primary and secondary structure elements. Dimers adopt a variable architecture, while their formation still results in a hibernating state. In vivo relevance of these findings and the reason for the dynamic association of the A49/A34.5related subunits are still under debate awaiting further investigation. Nevertheless, we conclude that the principles underlying Pol I regulation 'activation by contraction' and 'hibernation by dimerization' are conserved among organisms.

\section{Methods}

Construction of AC40-tagged S. pombe strain. A construct for genomic insertion of a 10xHis/Flag tag was ordered as plasmid (Gene Art). The construct was amplified and genomically inserted into the haploid S. pombe strain 972h-: A 100 $\mathrm{ml}$ YPD culture was started at optical density $\left(\mathrm{OD}_{600}\right)$ of 0.25 from an over night culture at $30^{\circ} \mathrm{C}$. After $5-6 \mathrm{~h}, \mathrm{OD}_{600}$ was at 1.0 and cells were harvested in $250 \mathrm{~m}$ conical tubes $(1361 \mathrm{~g}, 5 \mathrm{~min})$. Cells were resuspended in $25 \mathrm{ml}$ sterile water by vortexing and again centrifuged. Cells were resuspended in $1 \mathrm{ml}$ of sterile $100 \mathrm{mM}$ 
a

Pol I Monomer and EC structures are similar in Sp and Sc
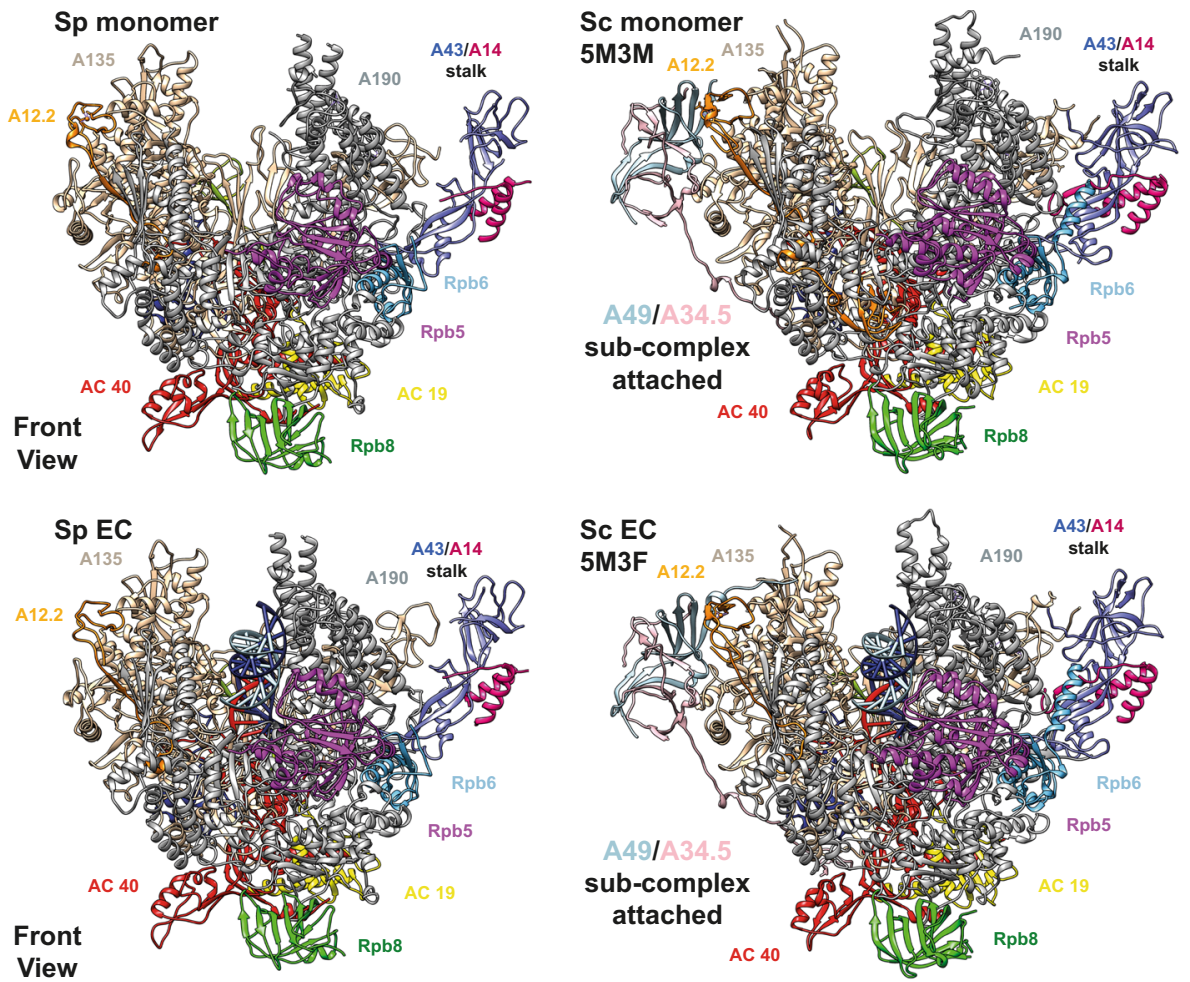

b

Pol I dimer structures diverge between Sp and Sc

S. pombe Pol I Dimer

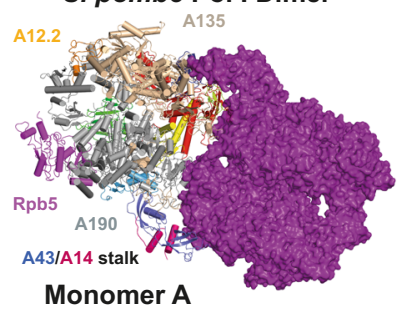

Monomer A

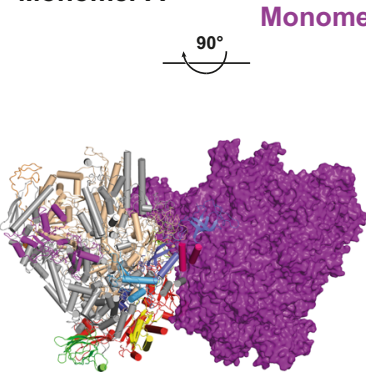

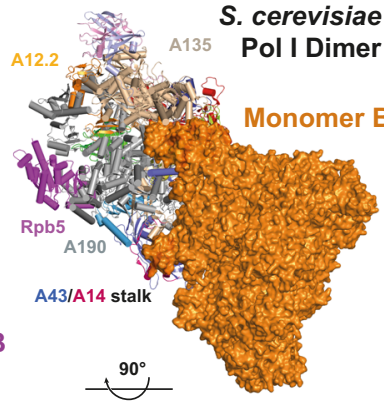
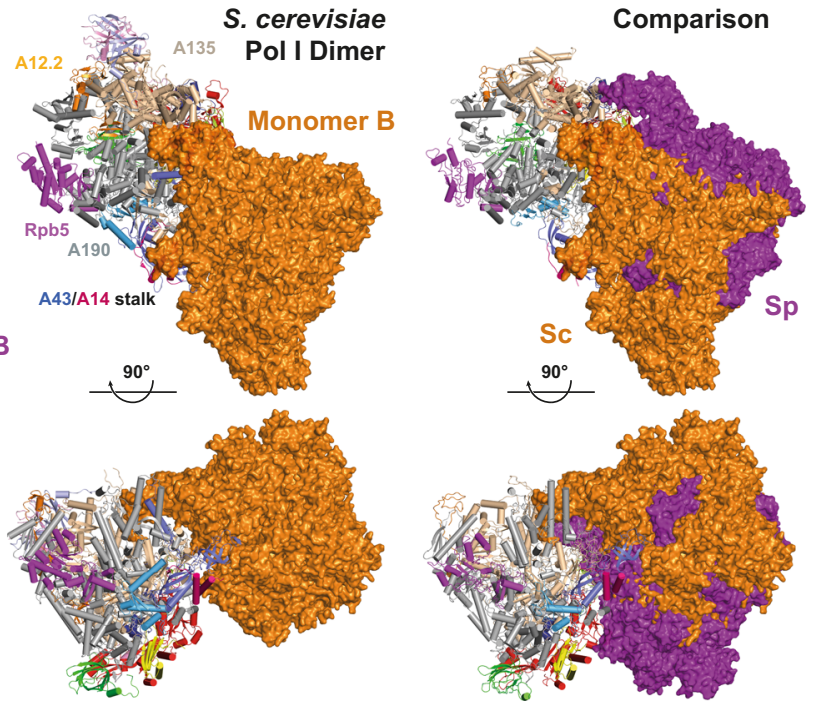

c Active center cleft expansion is conserved between Sc and Sp Pol I but most pronounced in Sc

\begin{tabular}{|c|c|c|c|}
\hline Organism & Structure & Downstream Cleft width $[\AA]]$ & Upstream Cleft width $[\AA]$ \\
\hline \multirow{3}{*}{ Saccharomyces cerevisiae } & Dimer $4 C 2 M$ & 42 & 39 \\
\hline & Monomer $5 M 3 M$ & 41 & 36 \\
\hline & EC $5 M 3 F$ & 34 & 25 \\
\hline \multirow{3}{*}{ Schizosaccharomyces pombe } & Dimer & 41 & 35 \\
\hline & Monomer & 40 & 35 \\
\hline & $\mathrm{EC}$ & 34 & 28 \\
\hline
\end{tabular}

Fig. 4 Comparison of Sc and Sp Pol I structures. a Structural comparison of Pol I monomers (top) and ECs (bottom) in Sp (left) and Sc (right) displays the similar architecture of both enzymes. Upon EC formation, both Pol I versions contract their active sites (Supplementary Movie 1). b Comparison of Sp and Sc Pol I dimers. First monomer (cartoon tubes) overlaid via subunit A135. Second monomer (space-filling; Sp purple, Sc orange) is attached via upstream face but globally shifted in location. Increased cleft expansion compared to monomers is observed in Sc but not in Sp. This is likely a consequence of stalk insertion into the active center cleft and formation of the connector helix at the clamp core domain of the second monomer in Sc. Compare Supplemental Movie 2. c Quantification of cleft widths in Sc and Sp Pol I structures. In Sc structures, upstream width measured between residues Arg 434 in subunit A135 and Val 418 in subunit A190, downstream between Gly 231 and Lys 1331 in subunit A190. In Sp structures, upstream width measured between the corresponding residues Arg 409 in subunit A135 and Arg 425 in subunit A190, downstream between Lys 226 and Ser 1338 in subunit A190. 
$\mathrm{Li}_{2} \mathrm{Ac}$ solution. The suspension was then transferred into a $1.5 \mathrm{ml}$ reaction tube and centrifuged for $15 \mathrm{~s}$ (tabletop centrifuge, full speed). Supernatant was removed and the pellet resuspended in $400 \mu \mathrm{l}$ of fresh $100 \mathrm{mM} \mathrm{Li}_{2} \mathrm{Ac}$. In parallel, $500 \mu \mathrm{l}$ of salmon sperm DNA $(2 \mathrm{mg} / \mathrm{ml})$ were boiled at $95^{\circ} \mathrm{C}$ for $5 \mathrm{~min}$ and quickly chilled on ice. The cells were then split into $100 \mu \mathrm{l}$ aliquots, pelleted and the supernatant removed. To a pellet, the following transformation mix was added in the following order: (1) $240 \mu \mathrm{l}$ sterile PEG3350 (50\% w/v), (2) $36 \mu \mathrm{l} 1 \mathrm{M} \mathrm{Li}_{2} \mathrm{Ac}$, (3) $50 \mu \mathrm{l} \mathrm{salmon}$ sperm DNA ( $2 \mathrm{mg} / \mathrm{ml})$, and (4) $34 \mu \mathrm{l}$ PCR product of the insertion construct. Tubes were vigorously vortexed for more than $1 \mathrm{~min}$ and incubated at $30^{\circ} \mathrm{C}$ for 30 min under shaking. Subsequently, reactions were transferred to $42{ }^{\circ} \mathrm{C}$ and incubated under shaking for $25 \mathrm{~min}$. Cells were then pelleted (tabletop centrifuge at $6000 \mathrm{~g}$ for $15 \mathrm{~s}$ ), the supernatant removed and cells were resuspended in $1 \mathrm{ml}$ YPD medium. Cells were transferred into $15 \mathrm{ml}$ conical tubes and shaken at $30^{\circ} \mathrm{C}$ for $3 \mathrm{~h}$. After centrifugation at $1361 \mathrm{~g}$ for $5 \mathrm{~min}$, the pellet was resuspended in $500 \mu \mathrm{l}$ sterile water and plated on YPD plates with Kanamycin/G418. The plates were incubated at $30^{\circ} \mathrm{C}$ for $3-4$ days, single colonies picked and re-plated on fresh plates. For verification of correct genomic insertion, the respective regions were amplified by PCR and sequenced.

Fermentation of S. pombe. S. pombe cells were plated on YPD plates and grown at $30^{\circ} \mathrm{C}$ for $48-72 \mathrm{~h}$. A preculture of $500 \mathrm{ml}$ was started and grown over night in YPD at $30^{\circ} \mathrm{C}$ under shaking. Cells were inspected for contaminations via light microscopy and secondary cultures of $2 \mathrm{l}$ each were inoculated at a starting $\mathrm{OD}_{600}$ of $0.3-0.5$. After $10-12 \mathrm{~h}$, cells were inspected visually and transferred into the $200 \mathrm{l}$ fermenter at a starting $\mathrm{OD}_{600}$ of $0.30-0.35$. YPD medium was prepared in the fermenter, but $\mathrm{pH}$ was not adjusted and was therefore at $\sim 6.0$ initially. The medium was autoclaved and Ampicillin and Tetracycline were added to final concentrations of $100 \mu \mathrm{g} / \mu \mathrm{l}$ and $12.5 \mu \mathrm{g} / \mu \mathrm{l}$, respectively. Antifoam reagent was added to reduce foaming during the fermentation. The fermenter was operated at $22 \mathrm{Nl} / \mathrm{min}$ (normal litres per minute) air influx and with $250 \mathrm{rpm}$ stirring at $30^{\circ} \mathrm{C}$. After $11-13 \mathrm{~h}$, an $\mathrm{OD}_{600}$ of 6.0 to 7.5 was reached and cells were harvested with a continuous-flow centrifuge, resuspended in freezing buffer (150 mM HEPES pH 7.8, $60 \mathrm{mM} \mathrm{MgCl}_{2}$, 20\% v/v glycerol, $5 \mathrm{mM}$ DTT, $1 \mathrm{mM}$ PMSF, $1 \mathrm{mM}$ Benzamidine, $60 \mu \mathrm{M}$ Leupeptin, $200 \mu \mathrm{M}$ Pepstatin; $0.5 \mathrm{ml}$ buffer for each g of cells) and flash-frozen in liquid nitrogen for storage at $-80^{\circ} \mathrm{C}$.

Pol I purification. The protocol for the purification of $S c$ Pol I ${ }^{5,45}$ was slightly modified to be applicable for 10x His tagged S. pombe Pol I

Frozen fermenter pellets $(=150 \mathrm{~g}$ cells in a total volume of $225 \mathrm{ml})$ were thawed and ammonium sulfate concentration adjusted to $400 \mathrm{mM}$. Cells were lysed after adding $3 \mathrm{ml} \mathrm{PI} \mathrm{(100x)} \mathrm{and} 200 \mathrm{ml}$ glass beads (diameter $0.5 \mathrm{~mm}$ ) by bead beating for $90 \mathrm{~min}$ (30 s mixing, $60 \mathrm{~s}$ break) under constant cooling. After cell lysis glass beads were removed by filtering and washed with dilution buffer (100 mM HEPES $\left.\mathrm{pH} 7.8,20 \mathrm{mM} \mathrm{MgCl} 2,400 \mathrm{mM}\left(\mathrm{NH}_{4}\right)_{2} \mathrm{SO}_{4}\right)$. The crude cell extract was then centrifuged $\left(4^{\circ} \mathrm{C} ; 8,600 \mathrm{~g}\right.$; JLA 16.250$)$ for $60 \mathrm{~min}$ to remove the cell debris. The supernatant was afterwards ultracentrifuged $\left(4^{\circ} \mathrm{C}, 167,424 \mathrm{~g} ; 45 \mathrm{Ti}\right.$ rotor) for 90 $\mathrm{min}$. The top fat layer was carefully removed using a $25-\mathrm{ml}$ pipette, the mid-layer was subsequently collected without disturbing the viscous bottom DNA-pellet. The aspired mid-layer was dialysed overnight $(16 \mathrm{~h}+)$ at $4{ }^{\circ} \mathrm{C}$ against dialysis buffer (50 mM KAc, $20 \mathrm{mM}$ HEPES pH 7.8, $1 \mathrm{mM} \mathrm{MCl}, 10 \%$ v/v glycerol, $10 \mathrm{mM} \beta-$ Mercaptoethanol, 1x PI (Benzamidine \& PMSF)). The dialysed extract was ultracentrifuged for $2 \mathrm{~h}\left(4^{\circ} \mathrm{C} ; 41,856 \mathrm{~g} ; 45 \mathrm{Ti}\right.$ rotor). The Pol I containing pellet was resuspended and pellets pooled in Res/W1 buffer (1.5 M KAc, $20 \mathrm{mM}$ HEPES $\mathrm{pH}$ $7.8,1 \mathrm{mM} \mathrm{MgCl} 2,10 \mathrm{mM}$ Imidazole, $10 \% \mathrm{v} / \mathrm{v}$ glycerol, $10 \mathrm{mM}$ ß-Mercaptoethanol, $0.5 \mathrm{PI})$. After $2 \mathrm{~h}$ incubation on a rotating wheel $\left(4^{\circ} \mathrm{C} ; 10 \mathrm{rpm}\right) 4 \mathrm{ml}$ equilibrated Ni-NTA beads were added to the suspension and further incubated for $4 \mathrm{~h}\left(4^{\circ} \mathrm{C}\right.$, $7 \mathrm{rpm})$. After incubation the suspension was decanted into gravity columns, the Pol I binding Ni-NTA beads were subsequently washed with Res/W1 buffer $(5 \mathrm{CV})$ and W2 buffer ( $300 \mathrm{mM} \mathrm{KAc,} 20 \mathrm{mM}$ HEPES pH 7.8, $1 \mathrm{mM} \mathrm{MgCl}, 25 \mathrm{mM}$ Imidazole, $10 \% \mathrm{v} / \mathrm{v}$ glycerol, $10 \mathrm{mM}$ B-Mercaptoethanol) (5 CV). Pol I was then eluted with $20 \mathrm{ml}$ total volume of E200 buffer ( $300 \mathrm{mM} \mathrm{KAc}, 20 \mathrm{mM}$ HEPES pH 7.8, $1 \mathrm{mM}$ $\mathrm{MgCl}_{2}, 200 \mathrm{mM}$ Imidazole, 10 \% v/v glycerol, $10 \mathrm{mM}$ B-Mercaptoethanol).

The eluate was therefore ultracentrifuged $\left(4^{\circ} \mathrm{C} ; 46,378 \mathrm{~g} ; 45 \mathrm{Ti}\right.$ rotor) for $20 \mathrm{~min}$ and loaded on a MonoQ 10/100 column (GE Healthcare) equilibrated with 15\% B (Mono-Buffer A: $20 \mathrm{mM}$ HEPES pH 7.8, $1 \mathrm{mM} \mathrm{MgCl}_{2}, 10 \%$ v/v glycerol, $5 \mathrm{mM}$ DTT; Mono-Buffer B: $2 \mathrm{M} \mathrm{KAc}, 20 \mathrm{mM}$ HEPES pH 7.8, $1 \mathrm{mM} \mathrm{MgCl}_{2}, 10 \% \mathrm{v} / \mathrm{v}$ glycerol, $5 \mathrm{mM}$ DTT). Pol I was eluted with a linear gradient of $13 \mathrm{CVs}$ from $0.3 \mathrm{M}$ to $1.4 \mathrm{M} \mathrm{KAc}$ (elution at around $0.9 \mathrm{M} \mathrm{KAc}$ ). Pol I containing fractions were pooled and diluted $200 \mathrm{mM} \mathrm{KAc}$ with Buffer A and again centrifuged $\left(4^{\circ} \mathrm{C} ; 16,696\right.$ g; 45Ti rotor). Next, the sample was loaded on a MonoS 5/50 column (GE Healthcare) equilibrated with $200 \mathrm{mM} \mathrm{KAc}$. Pol I was eluted with a linear gradient from $0.2 \mathrm{M}$ to $0.7 \mathrm{M} \mathrm{KAc}$ with a plateau of $5 \mathrm{CV}$ at $0.35 \mathrm{M}$ (elution at around $0.5 \mathrm{M}$ $\mathrm{KAc}$ ). The peak fractions were analyzed on a gel, pooled, concentrated (Amicon; $100 \mathrm{kDa}$ Molecular weight cut-off), flash-frozen in liquid nitrogen, and stored at $-80^{\circ} \mathrm{C}$.

RNA elongation and cleavage assays. Purified $S c$ or $S p$ Pol I ( $1,0.5$ or 0.25 pmol) was pre-incubated with 0.25 pmol of pre-annealed minimal nucleic acid scaffold (template DNA: 5'-CGAGGTCGAGCGTGTCCTGGTCTAG-3', non-template
DNA: 5'-CGCTCGACCTCG-3'; RNA: 5'-FAM-GACCAGGAC-3') in transcription buffer $\left(20 \mathrm{mM}\right.$ HEPES pH 7.8, $60 \mathrm{mM}\left(\mathrm{NH}_{4}\right)_{2} \mathrm{SO}_{4}, 8 \mathrm{mM} \mathrm{MgSO}_{4}, 10 \mu \mathrm{M}$ $\mathrm{ZnCl}_{2}, 10 \%$ (v/v) glycerol, $10 \mathrm{mM}$ DTT) for $20 \mathrm{~min}$ at $20^{\circ} \mathrm{C}$. For RNA elongation, NTPs (1.4 mM end concentration each) were added and the reaction was incubated for $30 \mathrm{~min}$ at $28^{\circ} \mathrm{C}$. To examine cleavage activity, the pre-incubated reaction with a twofold molar excess of Pol I compared to scaffold was incubated for $30 \mathrm{~min}$ at $28^{\circ} \mathrm{C}$ without the addition of NTPs. To stop the reaction an equal amount of $2 \mathrm{x}$ RNA loading dye ( $8 \mathrm{M}$ Urea, $2 \times$ TBE, $0.02 \%$ bromophenol blue, $0.02 \%$ xylene cyanol) was added and the sample was heated to $95^{\circ} \mathrm{C}$ for $5 \mathrm{~min}$. As control $0.25 \mathrm{pmol}$ of scaffold was treated identically, without the addition of polymerase and NTPs. 0.125 pmol of FAM-labeled RNA product (as well as a marker containing 9 nt, 15 nt and 21 nt long FAM-labeled RNAs: 5'-FAM-GACCAGGAC- $3^{\prime}$, 5'-FAM-AACGGAGACCAGGAC-3', 5'-FAM-UGUUCUUCUGGAAGUCCA GTT- $\left.3^{\prime}\right)$ was separated by gel electrophoresis ( $20 \%$ polyacrylamide gel containing $7 \mathrm{M}$ Urea) and visualized with a Typhoon FLA9500 (GE Healthcare).

Preparation of Pol I elongation complex. Synthetic DNA (IDT) and RNA (Qiagen) oligonucleotides were designed and assembled as described ${ }^{15}$, with the scaffold sequence for the template DNA (5'-AAGCTCAAGTACTTAAGCCTGGT CATTACTAGTACTGCC-3'), non-template DNA (5'-GGCAGTACTAGTAAAC TAGTATTGAAAGTACTTGAGCTT-3'), and RNA (5'-UAUCUGCAUGUAGAC CAGGC-3'; for the underlined nucleotides a methylene bridge between the $2^{\prime}-\mathrm{O}$ and the $4^{\prime}-\mathrm{C}$ of the ribose ring has been formed, thus creating a locked nucleic acid, LNA). Annealing was achieved by equimolar mixing $(40 \mu \mathrm{M})$, then heating to $95^{\circ} \mathrm{C}$, and gradually reducing the temperature to $20^{\circ} \mathrm{C}$ over $90 \mathrm{~min}$. Pol I $(1 \mathrm{mg} / \mathrm{ml})$ was incubated with a 1.35 -fold molar excess of pre-annealed EC-scaffold for $30 \mathrm{~min}$ at room temperature.

Crosslinking. Purified Pol I (Mono S Eluate at concentration $1.0-1.3 \mathrm{mg} / \mathrm{ml}$ ) was incubated with BS3 $\left(1 \mathrm{mM}\right.$ final concentration) for $30 \min \left(30^{\circ} \mathrm{C}, 300 \mathrm{rpm}\right)$, the reaction was stopped by adding Asp-Lys $\left(9 \mathrm{mM}\right.$ final; $\left.25^{\circ} \mathrm{C}, 300 \mathrm{rpm}\right)$ for $20 \mathrm{~min}$ followed by ammonium hydrogen carbonate $\left(60 \mathrm{mM}\right.$ final; $\left.25^{\circ} \mathrm{C} ; 300 \mathrm{rpm}\right)$ for $20 \mathrm{~min}$.

Cryo-EM grid preparation. The samples were centrifuged $\left(4^{\circ} \mathrm{C} ; 21,130 \mathrm{~g}\right.$; Eppendorf tabletop centrifuge) for $5 \mathrm{~min}$, to remove aggregates, and the supernatant carefully transferred into a fresh tube. The sample was then applied to a Superose 6 Increase 3.2/300 column in Solo4 buffer ( 5 mM HEPES pH 7.8, $1 \mathrm{mM}$ $\mathrm{MgCl}_{2}, 10 \mu \mathrm{M} \mathrm{ZnCl}_{2}, 150 \mathrm{mM} \mathrm{KCl}, 5 \mathrm{mM}$ DTT). The Pol I containing fraction was again centrifuged $\left(4^{\circ} \mathrm{C} ; 21,130 \mathrm{~g}\right)$ for $5 \mathrm{~min}$, and concentration was adjusted to approximately $100 \mu \mathrm{g} / \mathrm{ml}$. Four $\mu \mathrm{l}$ of sample was applied to a glow discharged (2x; $0.4 \mathrm{mbar} 15 \mathrm{~mA} ; 100 \mathrm{~s}) \mathrm{R} 1.2 / 1.3 \mathrm{Cu} \# 300$ grid (Quantifoil) and plunge frozen in liquid ethane (Vitrobot Mark IV, Thermo Fisher Scientific; $100 \%$ humidity; $4^{\circ} \mathrm{C}$; $5 \mathrm{~s}$ wait time; $5 \mathrm{~s}$ blotting time; blot force 12 ).

Single-particle cryo-EM. Images were collected on a Titan Krios Electron Microscope (Thermo Fisher Scientific) at $300 \mathrm{keV}$. Movies of 40 frames were acquired on a Falcon III direct electron detector at 75,000x magnification (pixel size $1.0635 \AA$ ). The movies were recorded in linear mode with a dose rate of $\sim 19 \mathrm{e}^{-} / \mathrm{px} / \mathrm{s}$ and a total dose of around $86 \mathrm{e}^{-} / \AA^{2}$. The defocus span from $-1.4 \mu \mathrm{m}$ to $-2.4 \mu \mathrm{m}$ alternating in $0.2 \mu \mathrm{m}$ intervals with a total of four exposures per hole.

Data processing. The EC dataset was processed using the RELION 3.0 suite ${ }^{46}$ (Supplementary Fig. 2). Movie frames were aligned and dose weighted using Relion's own implementation of MotionCor and Contrast Transfer Function (CTF) parameters were estimated using GCTF. A total of 3,598 movies were chosen based on accumulated motion, visual inspection and CTF values, astigmatism, defocus and maximal resolution. A set of 100 randomly picked micrographs throughout the dataset was chosen for reference-free auto-picking using the Laplacian-of-Gaussian (LoG) routine and yielding 2,829 particles. Two-dimensional classification resulted in templates for reference-based auto-picking yielding 299,038 particles. Two-fold binned particles (128 pixel boxes) were subjected to reference-free 2D classification (250 ̊ mask). Following removal of contaminants, a total of 156,493 unbinned particles were selected and aligned in 3D using an initial model generated in RELION as reference. These particles then underwent CTF refinement, bayesian polishing, followed by another round of CTF refinement. Masked Auto-refinement resulted in a reconstruction at $3.89 \AA$ overall resolution (0.143 FSC). Removal of particles showing increased flexibility of the Jaw and Clamp subdomains were removed by 3D Classification, resulting in 61,954 particles that allow reconstruction of an $S p$ Pol I EC at $4.00 \AA$ resolution.

The 'monomer' dataset was processed using the RELION 3.0 suite $^{46}$ unless stated otherwise (Supplementary Fig. 1). After importing pre-averaged movie frames (sums) the Contrast Transfer Function (CTF) parameters were estimated with the embedded Gctf program. Pre-processing was performed as described for the EC dataset. LoG picking resulted in a total of 11,594 particles from which 1,142 were chosen for template-based auto-picking following $2 \mathrm{D}$ classification. Removal of $\sim 90 \%$ of initially picked particles can be attributed to contamination and damage resulting from stress on the air-buffer interface. Initial auto-picking identified 874,753 particles from 4,333 micrographs, of which $\sim 50 \%$ were discarded as 
contamination based on 2D-Classification. A total of 477,791 particles were subjected to 3D classification using PDB 5M3M as reference. This allowed the removal of damaged particles and Pol I particles with highly flexible subdomains. The remaining 79,313 particles were subjected to CTF-refinement and bayesian polishing as for EC particles. The final 3D-reconstruction of monomeric Sp Pol I at a nominal resolution of $3.84 \AA$ shows an even orientational distribution of particles and some flexibility in the peripheral jaw, clamp and stalk regions.

In initial 2D classifications, minor dimer-classes were noticed. Thus, autopicked particles were re-extracted in larger boxes of 360 pixels and analyzed in a second, independent processing tree. From 510,315 Pol - like particles selected by $2 \mathrm{D}$ classification, a class of 17,552 particles could be attributed to well-defined dimers. Particles were centered on the interface between both Pols, re-extracted and another 450 poor particles removed based on 2D classification without sampling. Final 3D auto refinement imposing C2 symmetry yielded a reconstruction of $S p$ Pol I dimers at an overall resolution of $4.5 \AA$

Model building. At nominal resolutions of 3.8-4.0 ̊, we derive near-atomic models for most regions of Sp Pol I monomers and the EC. To commence model interpretation, we constructed homology models of subunits A190, A135, AC40, AC19, A43, A14 (ker1 in Sp) and A12.2 based on sequence comparison with their Sc homologs, alignment of actual and predicted secondary structures, and domain searches using HHPRED ${ }^{47}$. To construct homology models, the MODELLER ${ }^{48}$ implementation of UCSF Chimera was used ${ }^{49}$. Structures of the general subunits Rpb5, Rpb6, Rpb8, Rpb10 and Rpb12 were imported from the crystal structure of $S p$ Pol II ${ }^{50}$. Subdomain boundaries were defined based on $S c$ homology (Fig. 1 and Supplementary Fig. 4). Subdomains were then rigid body fitted into EC densities (which were first obtained) using COOT $^{51}$. While many regions allowed accurate fitting of sidechain orientations in the sharpened cryo-EM map, others suffered from poor main-chain tracing. Hence, density-guided modeling was performed in the clamp head, dock-insertion, foot-insertion and part of the jaw regions in subunit A190, as well as the subunit A12.2 and the toe domain of subunit AC40. Modeling of stalk-subunits A43 and A14 was limited to rigid body fitting of trimmed homology model domains in the unsharpened cryo-EM density. As a final step, real-space refinement was carried out using phenix.refine ${ }^{52}$. The Sp Pol I monomer was built by placement of the EC model and adjustment of subdomains in COOT, followed by manual inspection and real-space refinement using phenix. refine. The dimer model was constructed by placement of two monomers, rigid body fitting of subdomains and refinement in phenix-refine using NCS restraints.

Concentration-dependent dimerization using dynamic light scattering. Frozen Sp Pol I was thawed and diluted $(1.5 \mu \mathrm{M}, 1.0 \mu \mathrm{M}, 0.75 \mu \mathrm{M}, 0.5 \mu \mathrm{M})$ in S600 buffer (10 mM HEPES pH 7.8, $1 \mathrm{mM} \mathrm{MgCl}, 0.01 \mathrm{mM} \mathrm{ZnCl} 2,5 \mathrm{mM}$ DTT, $1.5 \%$ (v/v) glycerol, and $0.6 \mathrm{M} \mathrm{KAc}$ ) to a final volume of $20 \mu \mathrm{l}$. Technical duplicates of $10 \mu \mathrm{l}$ of each Pol I concentration were loaded into glass capillaries (Prometheus NT.48 Series nanoDSF Grade Standard Capillaries) and each capillary mounted into a Prometheus Panta (NanoTemper Technologies $\mathrm{GmbH}$ ). Fourty consecutive DLS measurements of each capillary were taken at $75 \%$ LED power, 100\% Laser power and $15{ }^{\circ} \mathrm{C}$ (total measurements per condition: $n=80$ ). Calculation and visualization were carried out using GraphPad Prism version 8.0.1 for Windows, GraphPad Software, La Jolla California USA, www graphpad.com. The boxes extend from the 25 th to 75 th percentiles ${ }^{53}$. The whiskers in Fig. $2 \mathrm{~b}$ are drawn down to the 10 th percentile and up to the 90th. Points below and above the whiskers are drawn as individual points.

Analytical size exclusion chromatography. A total of $50 \mu \mathrm{g}$ of frozen $S p$ Pol I was thawed and diluted to $2.93 \mu \mathrm{M}$ with SEC buffer ( $5 \mathrm{mM}$ HEPES pH 7.8, $1 \mathrm{mM}$ $\mathrm{MgCl}_{2}, 10 \mu \mathrm{M} \mathrm{ZnCl}$, $5 \mathrm{mM}$ DTT, and 1.5 M KAc (S1500), $600 \mathrm{mM}$ (S600), or $300 \mathrm{mM}(\mathrm{S} 300))$ to a total volume of $30 \mu \mathrm{l}$. The sample was centrifuged $\left(4^{\circ} \mathrm{C}\right.$; $21,130 \mathrm{~g}$; Eppendorf tabletop centrifuge) for $5 \mathrm{~min}$, to remove aggregates, and the supernatant carefully transferred into a fresh tube. The sample was then applied to a Superose 6 Increase 3.2/300 column (GE Healthcare; flow $0.035 \mathrm{ml} / \mathrm{min} ; 50 \mu \mathrm{l}$ fractions) in the respective buffer (S1500, S600, or S300). Peak fractions (Fig. 2) were diluted and negatively stained. After each run, a $30 \mu \mathrm{l}$ mixture of marker proteins (Thyroglobulin $(669 \mathrm{kDa})$, B-Amylase $(223 \mathrm{kDa})$, Albumin $(66.5 \mathrm{kDa})$ Calmodulin $(29 \mathrm{kDa})$ was applied to the column for calibration in each buffer.

Negative staining, EM data collection and image processing. Analytical SEC peak fractions were diluted to $20 \%(\mathrm{v} / \mathrm{v})$ and $10 \%(\mathrm{v} / \mathrm{v})$ in their respective buffers and were centrifuged $\left(4^{\circ} \mathrm{C} ; 21,130 \mathrm{~g}\right.$; Eppendorf tabletop centrifuge) for $5 \mathrm{~min}$. Five $\mu \mathrm{l}$ of the samples were then applied to 400-mesh copper grids (G2400C; Plano) with a selfmade carbon film of $\sim 7 \mathrm{~nm}$ thickness (self-made). After $30 \mathrm{~s}$, grids were washed in $200 \mu \mathrm{lddH_{2 }} \mathrm{O}$ for $30 \mathrm{~s}$, and stained three times in $20 \mu \mathrm{l}$ saturated uranyl formate solution (30 s). After each step, excess liquid was removed with a filter paper. Images were collected on a JEOL 2100-F Transmission Electron Microscope operated at $200 \mathrm{keV}$ and equipped with TVIPS-F416 (4kx4k) CMOS-detector at 40,000x magnification (pixel size $2.7 \AA$ ) with alternating defocus $(-2.5$ to $-4.5 \mu \mathrm{m})$.

The images were processed using RELION 3.1 (see above). For the high-salt SEC peak fraction (S1500), a total of 90 out of 98 collected micrographs were analyzed. A set of 10 randomly chosen images was used to train and optimize the reference-free auto-picking using Laplacian-of-Gaussian (LoG) routine. These settings were then applied on all 90 images yielding 40,544 particles that were applied to reference-free 2D classification ( $380 \AA$ mask). After removal of junk, a total of 20,054 particles were classified into 16 classes.

For the shown low-salt SEC peak fraction (S300), 129 micrographs were analyzed. A set of 10 randomly chosen images was used to train and optimize the reference-free auto-picking using Laplacian-of-Gaussian (LoG) routine. These particles underwent selection based on 2D classification and 3D centering yielding 3,532 particles that were subsequently used as a template for a reference-based auto-picking from all 129 images resulting in 36,733 particles. After 3D centering using the filtered density of PDB $5 \mathrm{M} 3 \mathrm{M}$ as reference and removal of junk particles by $2 \mathrm{D}$ classification ( $380 \AA$ mask), 24,462 particles remained. The outcome of a 2D classification into 16 classes was then compared to the high-salt particles (Supplementary Fig. 5).

Reporting summary. Further information on research design is available in the Nature Research Reporting Summary linked to this article.

\section{Data availability}

The cryo-EM density of Sp Pol I monomer, dimer and EC have been deposited in the Electron Microscopy Data Bank under accession codes EMD-11840, EMD-11841 and EMD-11842, respectively. Coordinates of the Sp Pol I monomer, dimer and EC were deposited with the Protein Data Bank under accession codes 7AOC, 7AOD and 7AOE, respectively. The data underlying Fig. 2 and unprocessed gel scans are provided in a separate Source Data File. Further material can be obtained from the corresponding author upon reasonable request. Source data are provided with this paper

Received: 3 June 2020; Accepted: 5 January 2021; Published online: 03 February 2021

\section{References}

1. Klinge, S. \& Woolford, J. L. Ribosome assembly coming into focus. Nat. Rev Mol. cell Biol. 20, 116-131 (2019).

2. Engel, C., Neyer, S. \& Cramer, P. Distinct mechanisms of transcription initiation by RNA polymerases I and II. Annu. Rev. biophysics 47, 425-446 (2018).

3. Khatter, H., Vorländer, M. K. \& Müller, C. W. RNA polymerase I and III: similar yet unique. Curr. Opin. Struct. Biol. 47, 88-94 (2017).

4. Moss, T., Langlois, F., Gagnon-Kugler, T. \& Stefanovsky, V. A housekeeper with power of attorney: the rRNA genes in ribosome biogenesis. Cell. Mol. life Sci. 64, 29-49 (2007).

5. Engel, C., Sainsbury, S., Cheung, A. C., Kostrewa, D. \& Cramer, P. RNA polymerase I structure and transcription regulation. Nature 502, 650-655 (2013).

6. Fernández-Tornero, C. et al. Crystal structure of the 14-subunit RNA polymerase I. Nature 502, 644-649 (2013)

7. Kostrewa, D., Kuhn, C.-D., Engel, C. \& Cramer, P. An alternative RNA polymerase I structure reveals a dimer hinge. Acta Crystallogr. Sect. D Biol. Crystallogr. 71, 1850-1855 (2015).

8. Milkereit, P., Schultz, P. \& Tschochner, H. Resolution of RNA polymerase I into dimers and monomers and their function in transcription. Biol. Chem. 378, 1433-1443 (1997).

9. Bischler, N. et al. Localization of the yeast RNA polymerase I-specific subunits. EMBO J. 21, 4136-4144 (2002).

10. Pilsl, M. et al. Structure of the initiation-competent RNA polymerase I and its implication for transcription. Nat. Commun. 7, 12126 (2016).

11. Engel, C. et al. Structural Basis of RNA Polymerase I Transcription Initiation. Cell 169, 120-131.e22 (2017).

12. Han, Y. et al. Structural mechanism of ATP-independent transcription initiation by RNA polymerase I. eLife 6; https://doi.org/10.7554/eLife.27414 (2017).

13. Sadian, Y. et al. Structural insights into transcription initiation by yeast RNA polymerase I. EMBO J. 36, 2698-2709 (2017).

14. Tafur, L. et al. Molecular Structures of Transcribing RNA Polymerase I. Mol. cell 64, 1135-1143 (2016).

15. Neyer, S. et al. Structure of RNA polymerase I transcribing ribosomal DNA genes. Nature; https://doi.org/10.1038/nature20561 (2016).

16. Torreira, E. et al. The dynamic assembly of distinct RNA polymerase I complexes modulates rDNA transcription. eLife 6; https://doi.org/10.7554/ eLife.20832 (2017).

17. Fernández-Tornero, C. RNA polymerase I activation and hibernation: unique mechanisms for unique genes. Transcription 9, 248-254 (2018).

18. Darrière, T. et al. Genetic analyses led to the discovery of a super-active mutant of the RNA polymerase I. PLoS Genet. 15, e1008157 (2019). 
19. Bernecky, C., Herzog, F., Baumeister, W., Plitzko, J. M. \& Cramer, P. Structure of transcribing mammalian RNA polymerase II. Nature 529, 551-554 (2016).

20. Kuhn, C.-D. et al. Functional architecture of RNA polymerase I. Cell 131, 1260-1272 (2007).

21. Tafur, L. et al. The cryo-EM structure of a 12-subunit variant of RNA polymerase I reveals dissociation of the A49-A34.5 heterodimer and rearrangement of subunit A12.2. eLife 8; https://doi.org/10.7554/eLife.43204 (2019).

22. Nakagawa, K. et al. The fission yeast RPA51 is a functional homolog of the budding yeast A49 subunit of RNA polymerase I and required for maximizing transcription of ribosomal DNA. Genes Genet. Syst. 78, 199-209 (2003).

23. Blattner, C. et al. Molecular basis of Rrn3-regulated RNA polymerase I initiation and cell growth. Genes Dev. 25, 2093-2105 (2011).

24. Engel, C., Plitzko, J. \& Cramer, P. RNA polymerase I-Rrn3 complex at $4.8 \AA$ resolution. Nat. Commun. 7, 12129 (2016)

25. Peyroche, G. et al. The recruitment of RNA polymerase I on rDNA is mediated by the interaction of the A43 subunit with Rrn3. EMBO J. 19, 5473-5482 (2000).

26. Imazawa, Y., Hisatake, K., Nakagawa, K., Muramatsu, M. \& Nogi, Y. The fission yeast RPA21 subunit of RNA polymerase I: an evolutionarily conserved subunit interacting with ribosomal DNA (rDNA) transcription factor Rrn3p for recruitment to rDNA promoter. Genes Genet. Syst. 77, 147-157 (2002)

27. Imazawa, Y. et al. The fission yeast protein Kerlp is an ortholog of RNA polymerase I subunit A14 in Saccharomyces cerevisiae and is required for stable association of Rrn3p and RPA21 in RNA polymerase I. J. Biol. Chem. 280, 11467-11474 (2005).

28. Passmore, L. A. \& Russo, C. J. Specimen preparation for high-resolution cryoEM. Methods Enzymol. 579, 51-86 (2016).

29. Sadian, Y. et al. Molecular insight into RNA polymerase I promoter recognition and promoter melting. Nat. Commun. 10, 5543 (2019).

30. Geiger, S. R. et al. RNA polymerase I contains a TFIIF-related DNA-binding subcomplex. Mol. cell 39, 583-594 (2010).

31. Viktorovskaya, O. V., Appling, F. D. \& Schneider, D. A. Yeast transcription elongation factor Spt5 associates with RNA polymerase I and RNA polymerase II directly. J. Biol. Chem. 286, 18825-18833 (2011).

32. Merkl, P. E. et al. RNA polymerase I ( $\mathrm{Pol} \mathrm{I}$ ) passage through nucleosomes depends on Pol I subunits binding its lobe structure. J. Biol. Chem. 295, 4782-4795 (2020).

33. Beckouet, F. et al. Two RNA polymerase I subunits control the binding and release of Rrn3 during transcription. Mol. Cell. Biol. 28, 1596-1605 (2008).

34. Knutson, B. A., McNamar, R. \& Rothblum, L. I. Dynamics of the RNA polymerase I TFIIF/TFIIE-like subcomplex: a mini-review. Biochem. Soc. Trans. 48, 1917-1927 (2020).

35. Albert, B. et al. RNA polymerase I-specific subunits promote polymerase clustering to enhance the rRNA gene transcription cycle. J. Cell Biol. 192 277-293 (2011).

36. Vannini, A. \& Cramer, P. Conservation between the RNA polymerase I, II, and III transcription initiation machineries. Mol. Cell 45, 439-446 (2012)

37. Moorefield, B., Greene, E. A. \& Reeder, R. H. RNA polymerase I transcription factor Rrn3 is functionally conserved between yeast and human. Proc. Natl. Acad. Sci. USA 97, 4724-4729 (2000).

38. Miller, G. et al. hRRN3 is essential in the SL1-mediated recruitment of RNA Polymerase I to rRNA gene promoters. EMBO J. 20, 1373-1382 (2001).

39. Milkereit, P. \& Tschochner, H. A specialized form of RNA polymerase I, essential for initiation and growth-dependent regulation of rRNA synthesis, is disrupted during transcription. EMBO J. 17, 3692-3703 (1998).

40. Mayer, C., Zhao, J., Yuan, X. \& Grummt, I. mTOR-dependent activation of the transcription factor TIF-IA links rRNA synthesis to nutrient availability. Genes Dev. 18, 423-434 (2004).

41. Bodem, J. et al. TIF-IA, the factor mediating growth-dependent control of ribosomal RNA synthesis, is the mammalian homolog of yeast Rrn3p. EMBO Rep. 1, 171-175 (2000).

42. Cavanaugh, A. H. et al. Rrn3 phosphorylation is a regulatory checkpoint for ribosome biogenesis. J. Biol. Chem. 277, 27423-27432 (2002).

43. Zhao, J., Yuan, X., Frödin, M. \& Grummt, I. ERK-dependent phosphorylation of the transcription initiation factor TIF-IA is required for RNA polymerase I transcription and cell growth. Mol. Cell 11, 405-413 (2003).

44. Pilsl, M. \& Engel, C. Structural basis of RNA polymerase I pre-initiation complex formation and promoter melting. Nat. Commun. 11, 1206 (2020).

45. Engel, C. Purification of crystallization-grade RNA polymerase I from $S$. cerevisiae. Methods Mol. Biol. 1455, 85-97 (2016).

46. Zivanov, J. et al. RELION-3: new tools for automated high-resolution cryo-EM structure determination. eLife 7, e42166 (2018)
47. Zimmermann, L. et al. A completely reimplemented MPI bioinformatics toolkit with a new HHpred server at its core. J. Mol. Biol. 430, 2237-2243 (2018).

48. Webb, B. \& Sali, A. Comparative protein structure modeling using MODELLER. Curr. Protoc. Bioinformatics 54, 5.6.1-5.6.37 (2016).

49. Pettersen, E. F. et al. UCSF Chimera-a visualization system for exploratory research and analysis. J. Comput. Chem. 25, 1605-1612 (2004).

50. Spåhr, H., Calero, G., Bushnell, D. A. \& Kornberg, R. D. Schizosacharomyces pombe RNA polymerase II at 3.6-A resolution. Proc. Natl. Acad. Sci. USA 106, 9185-9190 (2009).

51. Emsley, P. \& Cowtan, K. Coot: model-building tools for molecular graphics. Acta Crystallogr. Sect. D. Biol. Crystallogr. 60, 2126-2132 (2004).

52. Liebschner, D. et al. Macromolecular structure determination using X-rays, neutrons and electrons: recent developments in Phenix. Acta Crystallogr. Sect. D. Struct. Biol. 75, 861-877 (2019).

53. Hyndman, R. J. \& Fan, Y. Sample quantiles in statistical packages. Am. Statistician 50, 361 (1996).

\section{Acknowledgements}

We thank Michael Pilsl, Herbert Tschochner, Achim Griesenbeck and Philipp Milkereit for help and discussion, and Mona Höcherl for technical assistance. We thank Astrid Bruckmann for mass spectrometry, Gerhard Lehmann and Nobert Eichner for IT support, Gernot Längst for assistance with DLS measurements, Ralph Witzgall for JEM2100F access, and Achilleas Frangakis and Utz Ermel for initial cryo-EM screening Cryo-EM data were collected at the cryo-EM facility of the University of Würzburg with support from Bettina Böttcher and Christian Kraft. This work was supported by Deutsche Forschungsgemeinschaft SFB 960 (TP-A8) and the Emmy-Noether Programm (DFG grant no. EN 1204/1-1 to CE).

\section{Author contributions}

F.H. carried out $S p$ Pol I purification and characterization, prepared cryo-EM grids and carried out sequence analysis. F.H. and C.E. processed cryo-EM data. J.D. carried out functional elongation/cleavage assays. P.B. and C.E. built $S p$ Pol I models. C.E. designed and supervised research, established strains and purification protocols and prepared the manuscript with input from all authors.

\section{Funding}

Open Access funding enabled and organized by Projekt DEAL.

\section{Competing interests}

The authors declare no competing interests.

\section{Additional information}

Supplementary information The online version contains supplementary material available at https://doi.org/10.1038/s41467-021-21031-8.

Correspondence and requests for materials should be addressed to C.E.

Peer review information Nature Communications thanks the anonymous reviewer(s) for their contribution to the peer review of this work. Peer reviewer reports are available.

Reprints and permission information is available at http://www.nature.com/reprints

Publisher's note Springer Nature remains neutral with regard to jurisdictional claims in published maps and institutional affiliations.

\begin{abstract}
(c) (i) Open Access This article is licensed under a Creative Commons AY Attribution 4.0 International License, which permits use, sharing, adaptation, distribution and reproduction in any medium or format, as long as you give appropriate credit to the original author(s) and the source, provide a link to the Creative Commons license, and indicate if changes were made. The images or other third party material in this article are included in the article's Creative Commons license, unless indicated otherwise in a credit line to the material. If material is not included in the article's Creative Commons license and your intended use is not permitted by statutory regulation or exceeds the permitted use, you will need to obtain permission directly from the copyright holder. To view a copy of this license, visit http://creativecommons.org/ licenses/by/4.0/
\end{abstract}

(C) The Author(s) 2021 